\title{
Citrullinemia type II
}

INSERM

\section{Source}

INSERM. (1999). Orphanet: an online rare disease and orphan drug data base.

Citrullinemia type II. ORPHA:247585

Citrullinemia type II is a severe subtype of citrin deficiency (see this term) characterized clinically by adult onset (20 and 50 years of age), recurrent episodes of hyperammonemia and associated neuropsychiatric symptoms such as nocturnal delirium, confusion, restlessness, disorientation, drowsiness, memory loss, abnormal behavior (aggression, irritability, and hyperactivity), seizures, and coma. 